Br Heart 7 1982; 48: 601-3

\title{
Anomalous low insertion of right superior vena cava
}

\author{
R M FREEDOM, M S SCHAFFER, R D ROWE \\ From the Division of Cardiology, Department of Paediatrics, and Department of Radiology, The Hospital for Sick \\ Children and the University of Toronto, Faculty of Medicine, Toronto, Ontario, Canada
}

SUMMARY A 7 year old boy with complex congenital heart disease had a disinctive chest radiograph, and venous angiography showed anomalous low insertion of the right superior vena cava.

Among patients with congenital heart disease, anomalies of the right superior vena cava are relatively uncommon. ${ }^{1-3}$ The usual anomalies are congenital absence, anomalous drainage of the right superior vena cava into the left atrium as an isolated anomaly, congenital saccular aneurysm, and idiopathic dilatation of the right superior vena cava. ${ }^{45}$

We report a rare anomaly of the right superior vena cava in a 7 year old boy with complex congenital heart disease who presented to The Hospital for Sick Children, Toronto. ${ }^{6}$

\section{Case report}

This child presented at 5 weeks of age with mild cyanosis and congestive heart failure. The electrocardiogram showed so-called coronary sinus rhythm with a normal $P R$ interval and negative $P$ waves in leads II, III, and aVF. The frontal qRS axis was $+80^{\circ}$ and biventricular hypertrophy was evident. The chest $x$-ray film indicated a widened mediastinum, cardiomegaly, and pulmonary plethora. The heart was left sided and there was situs solitus.

On cardiac catheterisation, the systemic saturation was $91 \%$ and the pulmonary artery pressure was at systemic level. Angiography showed a single left ventricle, an outlet chamber, and concordant ventriculoarterial connections. The atrial situs was normal with right sided superior and inferior vena cavae and a left superior vena cava connected to the coronary sinus. Antifailure treatment was given, but because of refractory congestive heart failure the child underwent pulmonary artery banding and ligation of the left superior vena cava.

Subsequent growth and development were excellent. Follow-up catheterisation at 7 years of age showed that systemic arterial oxygen saturation was $86 \%$. The pulmonary arterial pressure distal to the band was $13 / 8$ $\mathrm{mmHg}$ (mean $10 \mathrm{mmHg}$ ) and the peak systolic right ventricular pressure was equal to systemic pressure.
Ventricular angiography confirmed the anatomy and showed the pulmonary artery band. Because the chest $x$-ray was unusual (Fig. 1), contrast material was injected in the right superior vena cava (Fig. 2). This showed anomalous low insertion of the right superior vena cava to the morphological right atrium, with separate atrial insertions of the superior and inferior vena cava.

\section{Discussion}

In 1975, Takanashi and colleagues ${ }^{7}$ reported anomalous high insertion of the inferior vena cava in a patient with a common atrium. They suggested that this venous anomaly originated from developmental failure of the sinoatrial fold and subsequent incomplete differentiation of the sinus venosus and its tributaries. In our case, the mechanism appears to be developmental failure of the right sinoatrial fold and incomplete absorption of the right anterior cardinal vein into the right limb of the sinus venosus.

The diagnosis of this most uncommon anomaly could have been suspected from the plain chest $x$-ray. The vertical density to the right of the mediastinum was not typical of anomalous pulmonary venous drainage or the "scimitar" syndrome. Dupuis and colleagues $^{6}$ showed a very similar angiogram and described one patient in whom the right superior vena cava also assumed an extramediastinal course.

Absence of the brachiocephalic vein was suggested as the cause of an extramediastinal superior vena cava. ${ }^{6}$ Our experience suggests otherwise. In assessing systemic venous connections in hundreds of patients with congenital heart disease we have identified many cases with normal visceral and atrial situs and bilateral superior vena cavae with the left superior vena cava connecting to the coronary sinus without an intervening left brachiocephalic vein. Only the patient we are reporting now had such an abnormal plain chest $x$-ray or angiogram. 


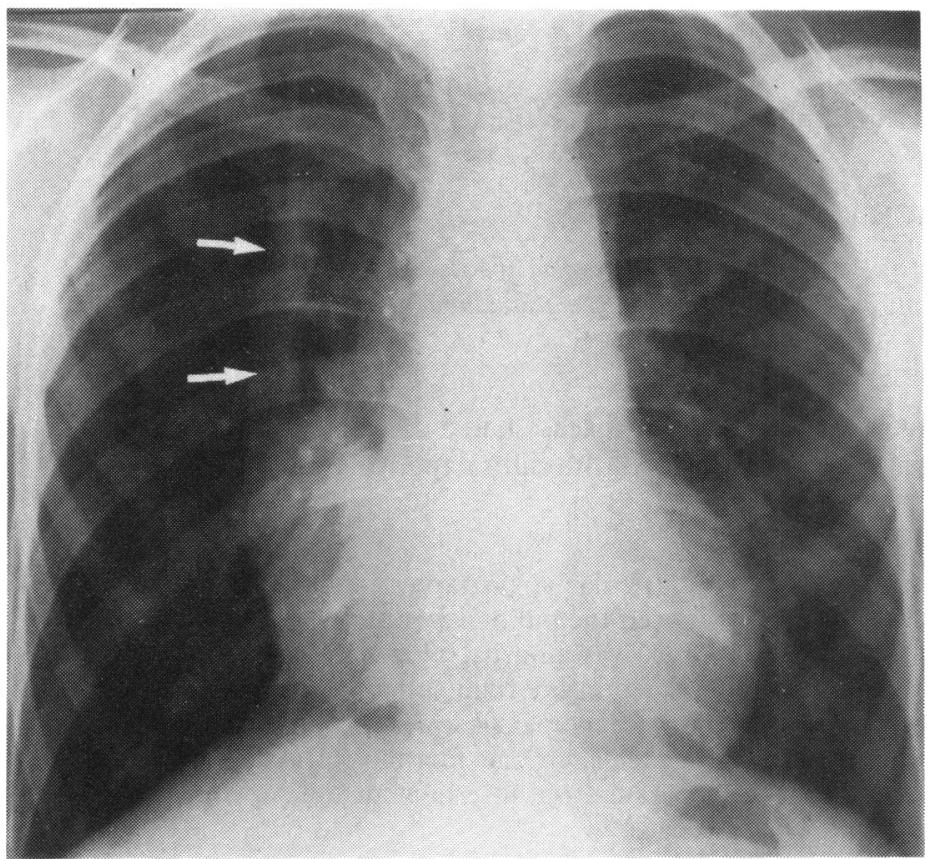

Fig. 1 Posteroanterior chest $\mathrm{x}$-ray showing a vertically orientated linear area of increased density in the right thorax (white arrow) surrounded by lung. The heart is mildly enlarged and centrally positioned in the chest; the pulmonary band is in place.

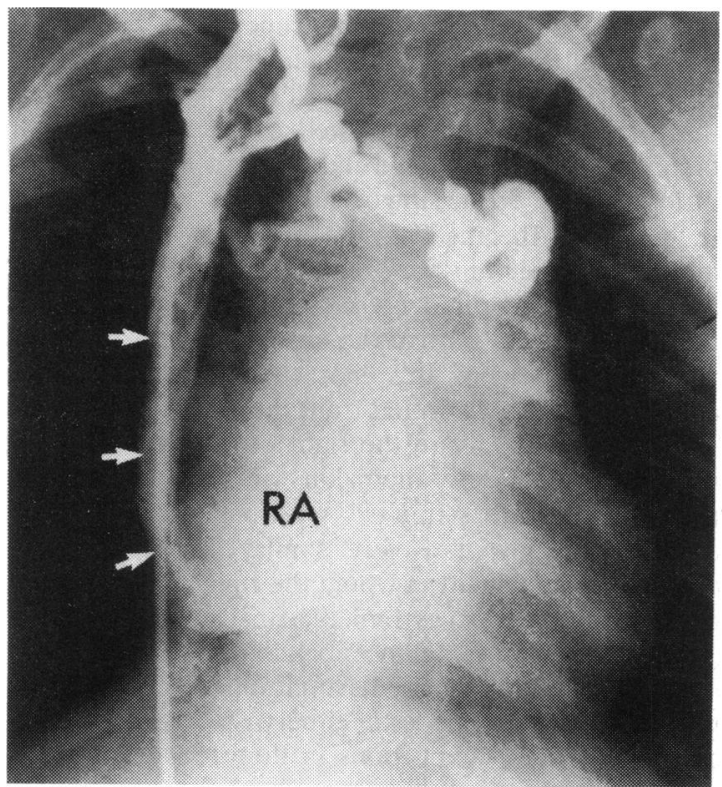

(a)

Fig. 2 (a) Posteroanterior frame from a cineangiocardiogram performed in the right superior vena cava showing anomalous low insertion of the right superior vena cava (white arrows) which connects to the right atrium $(R A)$ slightly superior to the insertion of the right sided inferior vena cava. (b) Lateral of this injection.

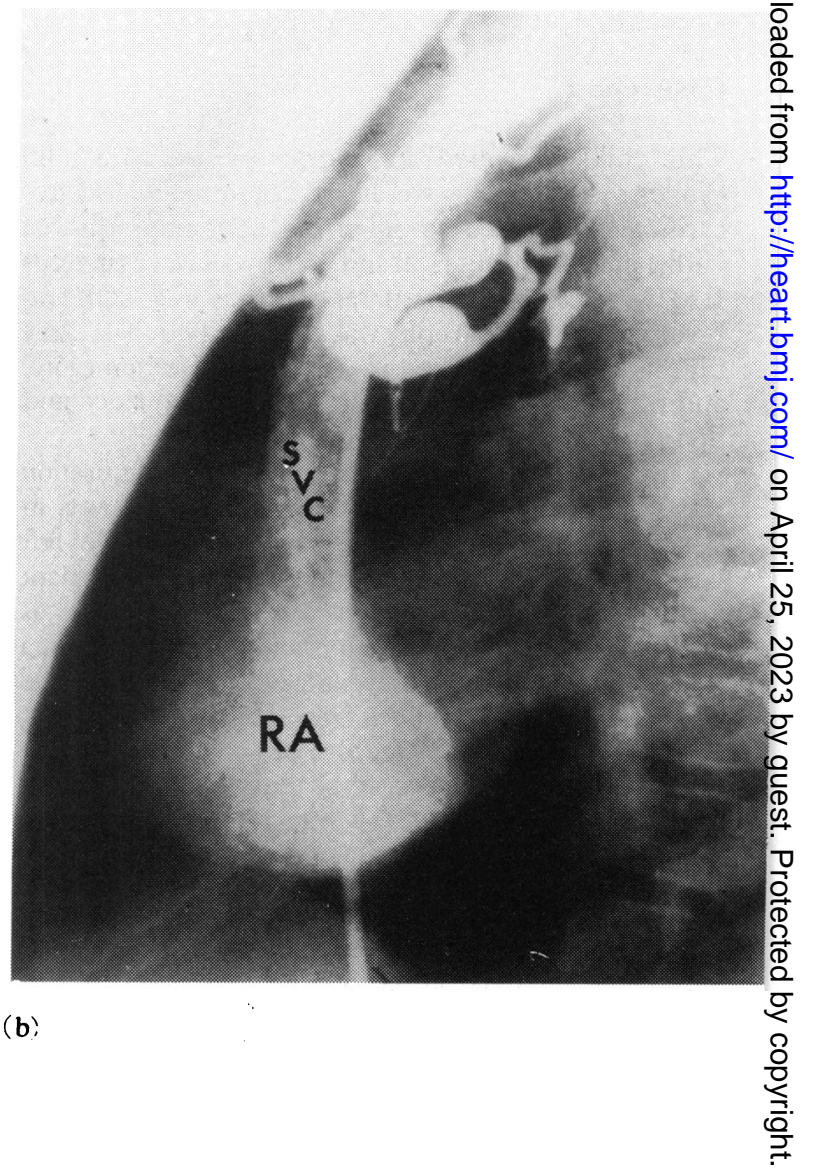


It is not surprising that our patient showed so-called coronary sinus rhythm on the surface electrocardiogram. ${ }^{89}$ Though this rhythm is rarely seen in normal subjects, it is common among patients with intrinsic abnormalities of the sinus venosus, for example those with sinus venosus atrial septal defect, or the polysplenia syndrome with azygos continuation of the inferior vena cava. This rhythm implies that the sinoatrial node is abnormal and the atrial pacemaker is ectopic. Electrophysiological studies indicate that the abnormal $P$ wave axis originates in the inferior left atrium or lower right atrium (including the orifice of the coronary sinus).

\section{References}

1 Braudo M, Beanlands DS, Trusler GA. Anomalous drainage of the right superior vena cava into the left atrium. Can . Med Assoc f 1968; 99: 715-9.

2 Lenox CC, Zuberbuhler JR, Park SC, et al. Absent right superior vena cava with persistent left superior vena cava: implications and management. Am $\mathcal{f}$ Cardiol 1980; 45: 117-22.

3 Modry DL, Hidvegi RS, LaFleche LR. Congenital saccular aneurysm of the superior vena cava. Ann Thorac Surg 1980; 29: 258-62.
4 Polansky S, Gooding CA, Potter B. Idiopathic dilatation of the superior vena cava (IDSVC). Pediatr Radiol 1974; 2: 167-74.

5 Vásquez-Pérez J, Frontera-Izquierdo P. Anomalous drainage of the right superior vena cava into the left atrium as an isolated anomaly. Rare case report. Am Heart f 1979; 97: 89-91.

6 Dupuis C, Risbourg B, Demougeot C. "Absence d'amarrage" de la veine cave supérieure au médiastin. A propos de 2 observations. Arch Mal Coeur 1977; 70: 415-7.

7 Takanashi Y, Anzai N, Okada T, Sano A, Ando M, Konno $\mathrm{S}$. Common atrium associated with anomalous high insertion of the inferior vena cava. $\mathcal{F}$ Thorac Cardiovasc Surg 1975; 69: 912-8.

8 van der Horst RL, Gotsman MS. Abnormalities of atrial depolarization in infradiaphragmatic interruption of inferior vena cava. Br Heart $\mathcal{F}$ 1972; 34: 295-300.

9 Freedom RM, Ellison RC. Coronary sinus rhythm in the polysplenia syndrome. Chest 1973; 63: 952-8.

Requests for reprints to Dr Robert M Freedom, Department of Cardiology, The Hospital for Sick Children, 555 University Avenue, Toronto, Ontario, Canada M5G 1 X8. 